\title{
An investigation of plasma and salivary oxytocin responses in breast- and formula-feeding mothers of infants
}

\author{
KAREN M. GREWEN ${ }^{\mathrm{a}}$, RUSSELL E. DAVENPORT ${ }^{\mathrm{a}}$, and KATHLEEN C. LIGHT ${ }^{\mathrm{b}}$ \\ aDepartment of Psychiatry, University of North Carolina at Chapel Hill, Chapel Hill, North \\ Carolina, USA

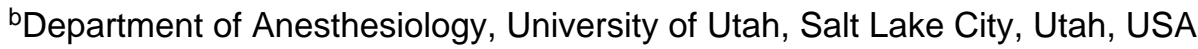

\section{Abstract}

Oxytocin (OT) is a peptide increasingly studied in relation to human social interactions, affiliation, and clinical disorders. Studies are constrained by use of invasive blood draws and would benefit from a reliable salivary OT assay. Our goals were to examine feasibility of salivary OT measurement, compare salivary to plasma OT responses in 12 breast- and 8 formula-feeding mothers, and assess the degree of correlation between plasma and salivary OT. Using a commercial EIA kit, we measured OT in 5 saliva and 7 plasma samples in a protocol designed to elicit changes in OT (Rest, Infant Interaction, Stress, Feeding). Breast-feeders had higher OT levels than formula-feeders across all conditions in plasma $(+36 \%)$ and saliva $(+23 \%)$. OT levels and ranges were similar in saliva and plasma, with slightly greater variance in saliva. Concurrently sampled plasma and salivary OT were correlated at end of Baseline Rest $(r=+.59, p=.022)$ and Post-Stress Recovery $(r=+.59, p=.025)$. These data suggest that salivary OT assay is feasible, and will be of value where plasma samples are not possible. Validation with larger samples is needed.

\section{Descriptors}

Oxytocin; Salivary oxytocin; Breast-feeding

\begin{abstract}
Oxytocin (OT) is a mammalian neuropeptide that has increasingly garnered the interest of researchers investigating biological correlates of human social interactions and relationships (Baumgartner, Heinrichs, Vonlanthen, Fischbacher, \& Fehr, 2008; Domes, Heinrichs, Michel, Berger, \& Herpertz, 2007; Grewen, Girdler, Amico, \& Light, 2005; Holt-Lunstad, Birmingham, \& Light, 2008; Light, Grewen, \& Amico, 2005; Neumann, 2008; Taylor et al., 2000), as well as mechanisms involved in social deficits present in psychiatric disorders including autism (Bartz \& Hollander, 2008; Carter, 2007; Hollander et al., 2007; Jacob et al., 2007; Meyer-Lindenberg, 2008; Posey, Erickson, \& McDougle, 2008), schizophrenia (Goldman, Marlow-O'Connor, Torres, \& Carter, 2008; Keri, Kiss, \& Kelemen, 2008), depression (Cyranowski et al., 2008), and substance abuse (Light et al., 2000, 2004). Understanding the physiology underlying normal and aberrant social interactions is important because epidemiological evidence highlights a key role for social relationships in health and disease, and suggests that more intimate, positive social affiliations may protect and prolong good health (Berkman, 1995; Kiecolt-Glaser \& Newton, 2001). Moreover, promising new studies suggest that OT administration may attenuate some of the social
\end{abstract}

Copyright (@ 2010 Society for Psychophysiological Research

Address reprint requests to: Karen M. Grewen, Ph.D., University of North Carolina at Chapel Hill, CB\#: 7175, Medical School Wing D, Chapel Hill, NC 27599-7175. karen_grewen@med.unc.edu. 
deficits that characterize clinical disorders (Bartz \& Hollander, 2008; Hollander et al., 2007; Meyer-Lindenberg, 2008), and may increase prosocial behaviors in healthy volunteers (Domes et al., 2007; Heinrichs \& Domes, 2008).

OT has been hypothesized as a physiological mediator of an integrated 'anti-stress' response attributable to social affiliation, with resulting long-term health benefits (Knox \& UvnasMoberg, 1998; Uvnas-Moberg, 1998a, 1998b). OT is produced primarily in the hypothalamus, where it is released into the central and peripheral circulations to coordinate, integrate, and modulate physiological, cognitive, and affective responses. Long known for the role it plays in lactation, uterine contraction, and reproductive behaviors, OT has also been linked to health-promoting cardiovascular (Diaz-Cabiale et al., 2000; Holst, UvnasMoberg, \& Petersson, 2002; Knox \& Uvnas-Moberg, 1998; Petersson, Lundeberg, \& Uvnas-Moberg, 1999), metabolic (Bjorkstrand, Hulting, Meister, \& Uvnas-Moberg, 1993; Petersson, Hulting, Andersson, \& Uvnas-Moberg, 1999; Uvnas-Moberg, 1994), analgesic (Grewen, Light, Mechlin, \& Girdler, 2008; Uvnas-Moberg, Bruzelius, Alster, \& Lundeberg, 1993), anti-inflammatory (Petersson, Wiberg, Lundeberg, \& Uvnas-Moberg, 2001) and anxiolytic (Blume et al., 2008) effects.

Importantly, experimental evidence in animals and humans relate OT to social bonds and interactions. Early animal studies, using more invasive and controlled methods, identify OT release and receptor distribution in the brain as important for monogamous pair-bonding (Williams, Carter, \& Insel, 1992), social recognition, social memory (Ferguson, Aldag, Insel, \& Young, 2001; Ferguson et al., 2000) and response to positive pleasant social stimuli (tactile, auditory, taste). Rodent, ungulate, and non-human primate models highlight the vital role of OT in initiation of maternal behaviors (Carter, 1998; Insel, 2003) and early motheroffspring interactions essential to offspring survival. In comparison, experimental studies in humans are generally confined to less invasive peripheral plasma assays. Nevertheless, these studies confirm that alterations in peripheral OT can be discerned in response to social affiliative stimuli (Gonzaga, Turner, Keltner, Campos, \& Altemus, 2006; Grewen et al,, 2005; Holt-Lunstad, et al., 2008; Light et al., 2005) and aspects of social relationships (Taylor et al., 2006).

Measurable increases in plasma OT elicited by breast-feeding (Altemus, Deuster, Galliven, Carter, \& Gold, 1995; Altemus et al., 2001; Carter, Altemus, \& Chrousos, 2001) make breast- and formula-feeding mothers an attractive comparison for investigations of OT correlates of psychosocial states and reactivity. Animal models support a role for OT in reductions in stress reactivity in mothers during lactation, including reductions in hypothalamic-pituitary-adrenal (HPA) (Windle et al., 1997a, 1997b) and fear responses to stressors (Carter \& Altemus, 1997). Whether lactation lowers anxiety and stress responsivity in human mothers is still in question. Altemus et al. reported that lactation was associated with reduced adrenocorticotropin hormone (ACTH), cortisol and glucose response to physical exercise, decreased basal levels of norepinephrine, and reduced systolic blood pressure (SBP) across all conditions in breast-feeding mothers (Altemus et al., 1995). Notably, this same group found no difference between breast- and formula-feeders in OT overall or in response to psychosocial stressors using the Trier Social Stress Test (TSST), but did report greater vagal cardiac control in breast-feeding mothers (Altemus et al., 2001). Consistent with these findings, our group reported no difference in blood pressure and heart rate responses to experimental stressors (speech and cold pressor) but did see smaller and less prolonged increases in vascular resistance (VR) responses in breast- compared with formula-feeding mothers, and higher plasma OT was correlated with smaller VR reactivity. In contrast to Altemus and colleagues, we reported a trend for greater OT concentrations across the protocol (Grewen \& Light, 2006), and greater likelihood of OT increases in 
response to infant contact in breast-feeding compared with formula-feeding mothers (Light et al., 2000).

Because human plasma assays necessitate invasive methods, OT measures are often limited by these technical requirements and are confined to clinical settings where blood sampling can be conducted by personnel trained, at the very least, in phlebotomy methods. In addition, plasma OT concentrations obtained by individual blood draws are likely to be contaminated by a stress-induced OT increase in response to the stress of the needle stick. Thus, an indwelling venous catheter and long habituation period prior to sampling are necessary to measure true resting levels and to maximize changes elicited solely by experimental manipulation. These difficulties make less invasive measurement of OT in saliva an attractive alternative. Although the physiological role of oxytocin in saliva is not entirely clear, measurement of OT, as with other commonly measured salivary endocrine products including cortisol and alpha amylase, would be a valuable tool to noninvasively assess changes in this neurohormone in clinical and nonclinical settings. Recent studies have documented detectable concentrations of OT in saliva using an earlier enzyme immunoassay (EIA) method without extraction (White-Traut et al., 2009), or using the sample method described here (Holt-Lunstad, 2008). In each of these studies, saliva was collected in subjects' own homes and concurrent plasma was not sampled, so it is, at present, unclear whether salivary OT will accurately reflect plasma concentrations.

Therefore, the three primary goals of this study were: 1) To examine the feasibility of noninvasive measurement of OT in human subjects (mothers of infants) using a standard kit assay with extraction; 2) To determine whether salivary OT would show increases and decreases to a variety of behavioral conditions that parallel changes evident in plasma OT, and would be equally good at differentiating responses from formula-feeding and breastfeeding mothers; 3 ) To assess the degree of correlation between the two measures during a limited number of conditions where both saliva and plasma samples were obtained concurrently or within a short temporal window of each other.

\section{Methods}

Participants

Twenty healthy mothers ( 12 who reported breast-feeding for $90 \%$ or more of feedings, 8 who reported formula-feeding for $80 \%$ or more of feedings) were recruited from the community during the perinatal period so that testing could be conducted within the window of 1-3 months postpartum. Exclusion criteria included self-report of prescription drug use, recent history of major depressive disorder and other psychological disorders, history of chronic disease in mother or infant, multiple birth, low birth weight (less than 4.5 pounds), gestational age less than 36 or greater than 42 weeks.

\section{Protocol}

All procedures were approved by the university institutional review board and all participants underwent informed consent before testing. Mothers brought their infants to the lab between 12:30-2:00 pm. Mothers were instrumented with cardiovascular electrodes for measurement of blood pressure and cardiac function (results not reported), and a flexible indwelling catheter (BD Saf-T-Intima, Becton Dickinson, Sandy, UT) was inserted into an antecubital vein, with saline drip set at $0.25-1.00$ drops per second to maintain lumen patency. A habituation period of at least $30 \mathrm{~min}$ followed before start of testing. During this time, subjects rested or sat quietly while blood pressure measurements were taken to calibrate cardiovascular equipment and were allowed to feed their infants $a d$ lib. During the habituation period prior to testing, 7 women breast-fed and 2 women formula-fed their 
infants. The remaining 11 mothers declined to feed (5 breast, 6 formula), saying that their infants had fed within the past hour (before leaving home).

The protocol consisted of the following events in fixed order: Baseline Rest (7 min), Mother-Infant Video-Taped Interaction (5 min), Post-Interaction Rest (7 min), Speech Task Stressor (8 min), Forehead Cold Pressor (2 min), Post-Stress Recovery (7 min), Infant Feeding. Plasma and salivary OT samples were taken concurrently immediately following baseline and post-interaction rests, and immediately following post-stress recovery. Plasma was sampled after 10 min of feeding, and saliva was sampled immediately after feeding (time varied between 10 and $19.5 \mathrm{~min}$ ). Figure 1 shows the timing of the experimental conditions and of plasma and salivary assays. Baseline Rest and Post-Task Recovery Periods: Mothers were instructed to sit quietly and relax as much as possible without falling asleep. A research assistant cared for the infant during this time but mothers were able to have infants near and to be in control of infant care at all times in order to prevent stressinduced OT responses during these periods. Mother-Infant Interaction (INXN): Infants were seated in an infant seat directly in front of mother for the 5-min face-to-face mother-infant interaction, while mother talked, touched, and played with her infant $a d$ lib. Maternal Speech Stress: Mothers were asked to give a short speech about a recent interpersonal event that caused feelings of anger or stress. This included $2 \mathrm{~min}$ of silent preparation, $3 \mathrm{~min}$ of active speaking (SPC), 3 min of passive listening to replay of audio-taped speech (REP). Forehead Cold Pressor Stress (ICE): Aplastic bag of ice and water $\left(\sim 4^{\circ} \mathrm{C}\right)$ was held on the forehead for $2 \mathrm{~min}$. Infant Feeding (FEED): Mothers were instructed to feed infants for 10 min following the post-stress recovery period. There were no specific instructions save to feed the infant for at least $10 \mathrm{~min}$. If the infant finished feeding before that time, we requested that mothers continue to hold their infants for the remainder of these $10 \mathrm{~min}$. At minute 10, plasma was sampled from the IV catheter which was obscured from mothers' view by a curtain. Mothers were allowed to continue feeding after the $10 \mathrm{~min}$ if they desired. Nineteen of 20 mothers fed for at least $10 \mathrm{~min}$, while one mother fed for approximately 5 $\mathrm{min}$. Mothers fed their infants during this feeding period (and during the habituation period) consistent with their feeding status categorization (breast-feeding mothers breast-fed their infants during the lab, formula-feeders fed with formula during the lab).

\section{Measurements}

Plasma was sampled for OT concentration at 7 time points: Baseline minute 7, Interaction minute 5 , Post-Interaction minute 7 , Speech minute 1.5 , Replay minute 1.5 , Recovery minute 7, Feeding minute 10. Saliva was sampled for OT assay at 5 time points: Baseline minute 7 , Post-Interaction minute 7, Ice minute 2, Recovery minute 7, Feed at completion (ranging from minute 10-minute 20).

Sample collection-For plasma OT, venous blood samples were collected into prechilled ethylenediaminetetraacetic acid (EDTA) tubes $(1 \mathrm{mg} / \mathrm{mL}$ blood) and kept cold during every step of processing. Because of the short half-life of OT peptide (1-6 min), the protease inhibitor Aprotinin (500 KIU/mL blood) was added to each tube to attenuate metabolic breakdown of peptide, as per strong recommendation by Assay Designs (Ann Arbor, MI) for this EIA. Samples were immediately cold-centrifuged at $1600 \times \mathrm{g}$ for $15 \mathrm{~min}$ at $4^{\circ} \mathrm{C}$. Plasma was then pipetted into chilled CryoTubes and immediately frozen at $-80^{\circ} \mathrm{C}$ until assay. For salivary OT, unstimulated passive drool samples were collected. Upon arrival to the lab visit, subjects were required to rinse their mouths thoroughly with water to remove any food particles. To standardize collection for each sample, subjects were asked to reserve saliva in their mouths without swallowing for $1 \mathrm{~min}$, which was timed with a stopwatch. After $1 \mathrm{~min}$ of saliva 'saving,' subjects used a straw to expectorate saliva into a pre-chilled $1.8 \mathrm{~mL}$ CryoTube, which was suspended in a cup of ice throughout the collection process. At least 1 
$\mathrm{mL}$ of saliva was required for each sample. If subjects could not produce this amount after 1 min, samples were maintained in the ice cup while subjects collected saliva in their mouths for an additional $1 \mathrm{~min}$. Saliva produced after the $2 \mathrm{nd}$ minute was then added to the CryoTube. CryoTubes were then immediately placed in a NUNC LabTop cooler (Thermoscientific, Rochester, NY) to flash freeze at $-20^{\circ} \mathrm{C}$ until protocol completion, when all samples were transferred to $-80^{\circ} \mathrm{C}$ until assay.

OT EIA-The method used to assay OT in saliva was similar to that specifically developed and validated for salivary OT by Carter et al. (2007). However, it should be noted that the current study used the latest Assay Designs OT EIA kit, which employs a different OT antibody than the one marketed by the same company prior to 2006, and kit instructions provided with this new antibody specify the need to perform an extraction step. Similar to the method used by Carter and others, which concentrated the sample 4.0 times using a dry down process (Bello, White-Traut, Schwertz, Pournajafi-Nazarloo, \& Carter, 2008; Carter et al., 2007; White-Traut et al., 2009), this required extraction step concentrates the sample 3.2 times. Notably, these differences in OT antibody and assay methods have resulted in marked differences in absolute OT levels and sensitivity reported. Plasma OT values are higher (15$300 \mathrm{pg} / \mathrm{ml}$ ) in studies using the older antibody without extraction, compared with those using this newer antibody $(2-40 \mathrm{pg} / \mathrm{mL}$ ) with the extraction step (Hoffman \& Brownley, 2009; Holt-Lunstad et al., 2008). The kit states that the sensitivity limit of the assay with the current OT antibody (without correcting for the concentration produced by the extraction process) is $11.6 \mathrm{pg} / \mathrm{mL}$, which is more than twice as high as with the different antibody provided with the Assay Designs kit prior to 2006. The result of the extraction procedure ensures that sample concentrations within the assay are above the assay sensitivity level and are detectable on the linear portion of the standard curve.

In the current study, all samples were assayed in duplicate, and the method of extraction of OT peptide was the same for both plasma and saliva samples, performed based on the instructions accompanying the currently available EIA kit. The first step was to equilibrate a strata-X $33 \mu \mathrm{m}$ polymeric reversed phase SPE sorbent in a 96-well plate containing $60 \mathrm{mg}$ sorbent per well (Phenomenex, Torrance, CA) by adding $1 \mathrm{ml} \mathrm{MeOH}$ followed by $1 \mathrm{ml}$ of water. The second step was to acidify $.8 \mathrm{ml}$ of plasma or saliva with $.4 \mathrm{ml}$ of $1.5 \%$ trifluoroacetic acid (TFA) and centrifuge at $6,000 \times \mathrm{g}$ for $20 \mathrm{~min}$ at $4^{\circ} \mathrm{C}$. Third, this supernatant was loaded onto the pre-treated strata-X plate, and the wells were slowly washed with $1.5 \mathrm{ml}$ of $0.1 \%$ TFA. Fourth, the peptide was eluted with $1 \mathrm{~mL}$ of $80 \%$ acetonitrile, then the eluant was collected in a polystyrene tube and evaporated to dryness under a nitrogen stream. Finally the residue was reconstituted in $250 \mathrm{ul}$ of assay buffer. The result of this extraction was to concentrate the sample 3.2 times, increasing precision and reducing matrix interference. Extraction efficiencies were determined by spiking a known amount of oxytocin into paired samples taken from homogenous pools of plasma and saliva created and stored for this purpose. Recovery of these known amounts allowed determination of OT extraction efficiencies of $96 \%$ for plasma and $93 \%$ for saliva.

OT levels in extracted plasma and saliva were then measured using the OT EIA, in which the endogenous OT hormone competes for the OT antibody binding sites with added OT linked to alkaline phosphatase. After the overnight incubation at $4^{\circ} \mathrm{C}$, the excess reagents were washed away and the bound OT phosphatase was incubated with substrate. After $1 \mathrm{~h}$, this enzyme reaction (which generates a yellow color) was stopped and the optical density (OD) was read on a Sunrise plate reader (Tecan, Research Triangle Park, NC) at $405 \mathrm{~nm}$. The intensity of the color is inversely proportional to the concentration of OT in the sample. The hormone content (in $\mathrm{pg} / \mathrm{ml}$ ) was determined by plotting the OD of each sample against a standard curve. After correcting for concentration produced by extraction, the lower limit of sensitivity was $2.0 \mathrm{pg} / \mathrm{ml}$. The intra- and inter-assay variations for this assay, which were 
practically validated and determined using a set control sample with known OT

concentration on each plate and in each run, were $4.8 \%$ and $8 \%$, respectively. Assay Designs reports cross-reactivity for similar mammalian neuropeptides in sera at less than 0.001 .

\section{Statistical Analysis}

In the current sample, $3 \%$ of saliva samples fell at or below the minimum detection level of $2 \mathrm{pg} / \mathrm{mL}$ (range $0.55-1.98 \mathrm{pg} / \mathrm{ml}$ ), and were thus set at $2.0 \mathrm{pg} / \mathrm{ml}$. Blood samples available for assay decreased as the protocol progressed due to loss of IV access or hemolysis ( samples lost at baseline $=0$, post-interaction $=2$, replay $=3$, recovery $=4$ ) and our decision to maintain IV access during feeding in a subsample $(n=10)$. OT concentration distributions at each time point were not skewed or kurtotic and therefore are presented as untransformed and unadjusted raw values.

We used Proc Mixed (SAS, Cary, NC) for repeated measures analyses to test differences in group mean plasma and salivary OT levels between Breast and Formula-feeding mothers across the protocol. Between-group $t$-tests were used to test differences in group mean OT levels between Breast and Formula-feeding mothers at each measurement time point. Paired $t$-tests were used to test within-subject changes in plasma and salivary OT levels across consecutive experimental conditions. Pearson product moment correlations were used to test the association of plasma and salivary OT levels assayed at each individual condition (Baseline, Post-Interaction, Stress, Recovery, Feeding).

\section{Results}

Subject characteristics are displayed in Table 1. Mothers were healthy, with mean body mass index (BMI) of 27.15 at time of testing between 30-96 days postpartum. Women differed on self-reported race (10 African American (50\%), 10 White (50\%)), parity (12 multipara (60\%), 8 primipara (40\%)), and feeding method (12 Breast, 8 Formula). Infants (10 male, 10 female) were healthy (mean birth weight: 7.04 pounds; mean gestational age: 39.35 weeks), unmedicated and free of acute illness or chronic disease at time of testing.

\section{Plasma oxytocin concentrations varied across laboratory conditions}

Plasma OT levels varied between subjects and within-subjects across experimental conditions. Mean values, standard errors, and range are displayed in Table 2. Plasma OT levels decreased significantly from 5-min mother-infant interaction to post-interaction rest (Interaction: $4.86 \pm 0.49$, Post-Intx Rest: $4.16 \pm 0.53 \mathrm{pg} / \mathrm{mL}$; within-subjects paired $t=2.30$, $p=.034)$, and increased significantly from post-interaction rest to minute 1.5 of speech task (Rest: $4.16 \pm 0.53$, Speech: $4.82 \pm 0.56 \mathrm{Q} 11 \mathrm{pg} / \mathrm{mL} ; t=-2.67, p=.016$ ). Mean plasma OT level at baseline was significantly lower in the 6 formula-feeding mothers who did not feed their infants during the habituation period compared with the 2 formula-feeding mothers who did $(2.93 \pm 0.22$ vs. $5.86 \pm 1.31 \mathrm{pg} / \mathrm{mL} ; p=.05)$. In the breast-feeding mothers, however, baseline plasma OT did not differ between the 7 who did vs. the 5 who did not feed their infants during habituation $(5.79 \pm 1.00$ vs. $5.30+0.90 \mathrm{pg} / \mathrm{mL}$, respectively). Furthermore, no group differences between those mothers who fed vs. did not persisted during the other experimental conditions.

\section{Salivary oxytocin concentrations varied across laboratory conditions}

In the current sample, $7 \%$ of the total 100 saliva samples were either of insufficient volume or were too thick/viscous for reliable extraction and therefore could not be included in analyses, and other samples (averaging 2.5 samples per time period) were missing due to other methodological problems. Mean salivary OT concentrations, shown in Table 2, varied across subjects and within subjects as a function of experimental condition. Significant 
within-subject increases in salivary OT from baseline to post-interaction rest were observed (Base $4.67 \pm 0.47$, Post-Interaction: $5.62 \pm 0.62 \mathrm{pg} / \mathrm{mL}$, paired $t=-2.69, p=.0225$ ).

Salivary OT levels at any time during the protocol did not differ based on whether or not mothers fed their infants during the habituation period.

\section{Breast vs. Formula Feeding and Oxytocin}

Mean plasma OT levels, depicted in Figure 2A, were higher across all conditions in breastfeeding vs. formula-feeding mothers (significant main effect of Feeding group, Breast: 5.56 $\mathrm{pg} / \mathrm{mL}$, Formula: $\left.4.10 \mathrm{pg} / \mathrm{mL} ; F_{2,18}=80.02, p<.0001\right)$.

Breast-feeding mothers also demonstrated greater mean salivary OT concentrations across the protocol compared with formula-feeding mothers (significant main effect of feeding group, Breast: $5.51 \mathrm{pg} / \mathrm{mL}$, Formula: $4.48 \mathrm{pg} / \mathrm{mL} ; F_{2,18}=137.43, p<.0001$ ), depicted in Figure 2B.

\section{Correlations of salivary and plasma oxytocin concentration}

Plasma and salivary OT levels sampled immediately following resting Baseline were positively correlated $(r=+.59, p=.022)$ such that women with higher plasma OT also demonstrated higher salivary OT. Figure 3 depicts this unadjusted relationship in data in 15 subjects from whom we obtained complete saliva and plasma samples for this period.

Similarly, a significant correlation of salivary and plasma OT collected at post-stress recovery period was observed. Figure 4 depicts positively correlated plasma and saliva OT concentrations immediately following the 7-min recovery rest period in 14 mothers with available plasma and saliva data $(r=+.59, p=.025)$.

Our study was not designed to assess correlations between plasma and saliva samples after stressors or after feeding since sampling of saliva in those events occurred from 1-10 min after the collection of plasma. At post-interaction rest, although samples were obtained in close temporal association, the plasma and saliva OT levels were not correlated $(r=-.04, p$ $=.88)$.

\section{Discussion}

The first goal of the present investigation was to examine the feasibility of measuring noninvasive salivary OT in human subjects using a standard EIA kit assay with an extraction step to concentrate the sample and reduce matrix interference. The goal was successfully achieved since only $7 \%$ of the nearly 100 saliva samples were too thick and viscous or too small in quantity to complete this assay effectively, and only $3 \%$ of the saliva samples from 20 mothers of infants yielded values below the threshold of detection.

The second goal of the study was to determine whether salivary OT levels can differentiate responses between formula-feeding and breast-feeding mothers across a variety of behavioral events in a comparable manner to plasma OT levels. This goal was partially successful in that breast-feeding mothers demonstrated higher mean levels of both plasma $(+36 \%)$ and salivary $(+23 \%)$ OT across all sampling times. This protocol successfully elicited changes in maternal plasma and salivary OT levels in response to infant contact and maternal stress. Both salivary and plasma OT levels increased when measured after infant feeding compared with the previous post-stress resting recovery period; however, these increases were not statistically significant. The small number of plasma OT samples taken during this feeding session $(n=10)$ may have limited our power to see differences between breast- and formula-feeding mothers at this time point. Across each individual time point in the protocol, differences in mean OT levels between breast- and formula-feeding mothers 
tended to be greater, and the variances tended to be smaller for plasma OT compared with salivary OT. This suggests that larger cell sizes may be necessary to detect differences with the noninvasive salivary measure.

We also achieved partial success for the third goal of the study, which was to examine direct correlations between plasma and salivary OT measures during three behavioral events (after baseline rest, after recovery from mother-infant interaction, and after recovery from the two stressors) where both saliva and plasma samples were obtained at nearly the same times. For the baseline rest and the post-stressor recovery, the invasive and non-invasive OT measures were closely correlated (both $r \mathrm{~s}=+.59$ ), while during recovery after interacting with their infants, the two measures showed essentially no correlation. It is not clear why the two measures failed to correlate for this post-interaction rest when breast- and formula-feeders did differ in both plasma and saliva OT levels at this time point. However, plasma levels at this time had fallen from the level seen immediately after the mother-infant interaction ended, and it appears that the mean saliva levels after post-interaction recovery look more like the plasma levels from right after the interaction ended. Thus, saliva levels may show a greater lag time in terms of changes, and thus may more closely reflect plasma levels during stable periods when the subjects are not showing marked increases or decreases.

Unlike Altemus et al. (2001), who did not find differences in levels of plasma OT in breastand formula-feeding mothers overall or in response to stressors, we did find significantly higher OT levels in plasma across the protocol in breast-feeding mothers. Moreover, we also found greater mean levels of salivary OT across the protocol in breast-feeding mothers. However, although both plasma and salivary OT levels appeared to increase with infant feeding, these increases were not statistically significant. This is not inconsistent with the only previous investigation of salivary OT in breast-feeding mothers, which did not include a formula-feeding comparison group, and simply documented that salivary OT levels increased in a consistent manner during the 30-min period immediately before mothers began to breast-feed their infants (White-Traut et al., 2009).

This study has several limitations. The sample size of 20 was quite modest, and replication in a larger sample would be very appropriate, particularly given the variability associated with salivary OT. Some variation in baseline OT levels and related responses may have resulted because not all women elected to feed their infants during the habituation period just prior to baseline. This may have influenced the baseline OT levels in formula-feeding mothers, but did not appear to influence breast-feeding mothers, and did not affect OT during later sampling points in either group. In future studies, we plan to standardize the time since last feeding before testing. In addition, in this study breast-feeding infants had greater contact with mothers' skin and breast than formula-fed infants. In future studies, it may be useful to standardize by having all mothers feed "skin-to-skin." Finally, it would have been much better if we had been able to obtain plasma and saliva samples within a few minutes of each other under all testing conditions rather than just some of them.

Although this study showed that salivary OT will sometimes, but not always, demonstrate similar responses to those seen in plasma OT, this does not lessen the potential value of the less invasive measure. Under some circumstances, salivary OT may be the superior of the two measures, or even the only possible measure to obtain. These circumstances include testing of subjects who cannot or choose not to participate if their blood must be drawn (such as children), and assessments during activities where needles are impractical, such as at home and in other real life locations. As an example of the latter, Holt-Lunstad et al. (2008) showed that salivary OT samples obtained at home were increased in married couples when they had recently practiced warm listening touch and massage, and were modestly correlated with plasma levels taken 1 week earlier in the clinic. In regard to the 
former, we are now continuing to use the mother-infant protocol described here, but are also taking saliva samples for OT from the infants. We believe that the strengths and weaknesses of this salivary sampling method are not different for OT than for other commonly measured biomarkers such as salivary cortisol or alpha amylase.

In summary, this protocol successfully elicited changes in plasma and salivary OT concentrations among 20 mothers in response to infant contact and stress. Levels and changes in OT concentration differed across activities and between mothers using breast- vs. formula-feeding methods. Similar means and ranges for OT values in saliva and plasma were observed, and the two measures were correlated when obtained concurrently after baseline rest and post-stress recovery. Feasibility of measuring salivary OT was a partial success and warrants further investigation.

\section{Acknowledgments}

This study was funded by NIH grants K01DA099949, R01HL084222, and RR00046. Special thanks are given to Cheryl Walker and Chihiro Christmas for their expertise with plasma and saliva sample processing, and to Mala Elam, RN, Marisa DeBurkarte, Jay Gregg, Patty Kuo, Riti Shah, Noah Pagano, Nick Zerona, Nitisha Desai, Michael Addamo, Janna Williams, and Poorvi Oza for their valuable contributions to data collection, management and analysis.

\section{References}

Altemus M, Deuster PA, Galliven E, Carter CS, Gold PW. Suppression of hypothalmic-pituitaryadrenal axis responses to stress in lactating women. Journal of Clinical Endocrinology \& Metabolism. 1995; 80(10):2954-2959. [PubMed: 7559880]

Altemus M, Redwine LS, Leong YM, Frye CA, Porges SW, Carter CS. Responses to laboratory psychosocial stress in postpartum women. Psychosomatic Medicine. 2001; 63(5):814-821. [PubMed: 11573030]

Bartz JA, Hollander E. Oxytocin and experimental therapeutics in autism spectrum disorders. Progress in Brain Research. 2008; 170:451-462. [PubMed: 18655901]

Baumgartner T, Heinrichs M, Vonlanthen A, Fischbacher U, Fehr E. Oxytocin shapes the neural circuitry of trust and trust adaptation in humans. Neuron. 2008; 58(4):639-650. [PubMed: 18498743]

Bello D, White-Traut R, Schwertz D, Pournajafi-Nazarloo H, Carter CS. An exploratory study of neurohormonal responses of healthy men to massage. Journal of Alternative and Complementary Medicine. 2008; 14(4):387-394.

Berkman LF. The role of social relations in health promotion. Psychosomatic Medicine. 1995; 57(3): 245-254. [PubMed: 7652125]

Bjorkstrand E, Hulting AL, Meister B, Uvnas-Moberg K. Effect of galanin on plasma levels of oxytocin and cholecystokinin. NeuroReport. 1993; 4(1):10-12. [PubMed: 7680910]

Blume A, Bosch OJ, Miklos S, Torner L, Wales L, Waldherr M, Neumann ID. Oxytocin reduces anxiety via ERK1/2 activation: Local effect within the rat hypothalamic paraventricular nucleus. European Journal of Neuroscience. 2008; 27(8):1947-1956. [PubMed: 18412615]

Carter CS. Neuroendocrine perspectives on social attachment and love. Psychoneuroendocrinology. 1998; 23(8):779-818. [PubMed: 9924738]

Carter CS. Sex differences in oxytocin and vasopressin: Implications for autism spectrum disorders? Behavioral Brain Research. 2007; 176(1):170-186.

Carter CS, Altemus M. Integrative functions of lactational hormones in social behavior and stress management. Annals of the New York Academy of Sciences. 1997; 807:164-174. [PubMed: 9071349]

Carter CS, Altemus M, Chrousos GP. Neuroendocrine and emotional changes in the post-partum period. Progress in Brain Research. 2001; 133:241-249. [PubMed: 11589134] 
Carter CS, Pournajafi-Nazarloo H, Kramer KM, Ziegler TE, White-Traut R, Bello D, Schwertz D. Oxytocin: Behavioral associations and potential as a salivary biomarker. Annals of the New York Academy of Sciences. 2007; 1098:312-322. [PubMed: 17435137]

Cyranowski JM, Hofkens TL, Frank E, Seltman H, Cai HM, Amico JA. Evidence of dysregulated peripheral oxytocin release among depressed women. Psychosomatic Medicine. 2008; 70(9):967975. [PubMed: 19005082]

Diaz-Cabiale Z, Narvaez JA, Garrido R, Petersson M, Uvnas-Moberg K, Fuxe K. Antagonistic oxytocin/alpha2-adrenoreceptor interactions in the nucleus tractus solitarii: Relevance for central cardiovascular control. Journal of Neuroendocrinology. 2000; 12(12):1167-1173. [PubMed: 11106973]

Domes G, Heinrichs M, Michel A, Berger C, Herpertz SC. Oxytocin improves “mind-reading” in humans. Biological Psychiatry. 2007; 61(6):731-733. [PubMed: 17137561]

Ferguson JN, Aldag JM, Insel TR, Young LJ. Oxytocin in the medial amygdala is essential for social recognition in the mouse. Journal of Neuroscience. 2001; 21(20):8278-8285. [PubMed: 11588199]

Ferguson JN, Young LJ, Hearn EF, Matzuk MM, Insel TR, Winslow JT. Social amnesia in mice lacking the oxytocin gene. Nature Genetics. 2000; 25(3):284-288. [PubMed: 10888874]

Goldman M, Marlow-O'Connor M, Torres I, Carter CS. Diminished plasma oxytocin in schizophrenic patients with neuroendocrine dysfunction and emotional deficits. Schizophrenia Research. 2008; 98(1-3):247-255. [PubMed: 17961988]

Gonzaga GC, Turner RA, Keltner D, Campos B, Altemus M. Romantic love and sexual desire in close relationships. Emotion. 2006; 6(2):163-179. [PubMed: 16768550]

Grewen KM, Girdler SS, Amico J, Light KC. Effects of partner support on resting oxytocin, cortisol, norepinephrine, and blood pressure before and after warm partner contact. Psychosomatic Medicine. 2005; 67(4):531-538. [PubMed: 16046364]

Grewen, KM.; Light, KC. Breast feeding and higher oxytocin are related to lower vascular resistance reactivity to stress. 64th Annual Meeting of the American Psychosomatic Society; Boulder, CO. 2006.

Grewen KM, Light KC, Mechlin B, Girdler SS. Ethnicity is associated with alterations in oxytocin relationships to pain sensitivity in women. Ethnicity and Health. 2008; 13(3):219-241. [PubMed: 18568974]

Heinrichs M, Domes G. Neuropeptides and social behaviour: Effects of oxytocin and vasopressin in humans. Progress in Brain Research. 2008; 170:337-350. [PubMed: 18655894]

Hoffman E, Brownley KA. Plasma and salivary oxytocin values quantified by EIA with extraction. Personal communication, May 2009. 2009

Hollander E, Bartz J, Chaplin W, Phillips A, Sumner J, Soorya L, et al. Oxytocin increases retention of social cognition in autism. Biological Psychiatry. 2007; 61(4):498-503. [PubMed: 16904652]

Holst S, Uvnas-Moberg K, Petersson M. Postnatal oxytocin treatment and postnatal stroking of rats reduce blood pressure in adulthood. Autonomic Neuroscience. 2002; 99(2):85-90. [PubMed: 12241092]

Holt-Lunstad J, Birmingham WA, Light KC. Influence of a "warm touch” support enhancement intervention among married couples on ambulatory blood pressure, oxytocin, alpha amylase, and cortisol. Psychosomatic Medicine. 2008; 70(9):976-985. [PubMed: 18842740]

Insel TR. Is social attachment an addictive disorder? Physiology \& Behavior. 2003; 79(3):351-357. [PubMed: 12954430]

Jacob S, Brune CW, Carter CS, Leventhal BL, Lord C, Cook EH Jr. Association of the oxytocin receptor gene (OXTR) in Caucasian children and adolescents with autism. Neuroscience Letters. 2007; 417(1):6-9. [PubMed: 17383819]

Keri S, Kiss I, Kelemen O. Sharing secrets: Oxytocin and trust in schizophrenia. Social Neuroscience. 2008:1-7. [PubMed: 18633851]

Kiecolt-Glaser JK, Newton TL. Marriage and health: His and hers. Psychological Bulletin. 2001; 127(4):472-503. [PubMed: 11439708]

Knox SS, Uvnas-Moberg K. Social isolation and cardiovascular disease: An atherosclerotic pathway? Psychoneuroendocrinology. 1998; 23(8):877-890. [PubMed: 9924742] 
Light KC, Grewen KM, Amico JA. More frequent partner hugs and higher oxytocin levels are linked to lower blood pressure and heart rate in premenopausal women. Biological Psychology. 2005; 69(1):5-21. [PubMed: 15740822]

Light KC, Grewen KM, Amico JA, Boccia M, Brownley KA, Johns JM. Deficits in plasma oxytocin responses and increased negative affect, stress, and blood pressure in mothers with cocaine exposure during pregnancy. Addictive Behaviors. 2004; 29(8):1541-1564. [PubMed: 15451123]

Light KC, Smith TE, Johns JM, Brownley KA, Hofheimer JA, Amico JA. Oxytocin responsivity in mothers of infants: A preliminary study of relationships with blood pressure during laboratory stress and normal ambulatory activity. Health Psychology. 2000; 19(6):560-567. [PubMed: 11129359]

Meyer-Lindenberg A. Impact of prosocial neuropeptides on human brain function. Progress in Brain Research. 2008; 170:463-470. [PubMed: 18655902]

Neumann ID. Brain oxytocin: A key regulator of emotional and social behaviours in both females and males. Journal of Neuroendocrinology. 2008; 20(6):858-865. [PubMed: 18601710]

Petersson M, Hulting A, Andersson R, Uvnas-Moberg K. Long-term changes in gastrin, cholecystokinin and insulin in response to oxytocin treatment. Neuroendocrinology. 1999; 69(3): 202-208. [PubMed: 10087452]

Petersson M, Lundeberg T, Uvnas-Moberg K. Short-term increase and long-term decrease of blood pressure in response to oxytocin-potentiating effect of female steroid hormones. Journal of Cardiovasccular Pharmacology. 1999; 33(1):102-108.

Petersson M, Wiberg U, Lundeberg T, Uvnas-Moberg K. Oxytocin decreases carrageenan induced inflammation in rats. Peptides. 2001; 22(9):1479-1484. [PubMed: 11514032]

Posey DJ, Erickson CA, McDougle CJ. Developing drugs for core social and communication impairment in autism. Child and Adolescent Psychiatric Clinics of North America. 2008; 17(4): 787-801. viii-ix. [PubMed: 18775370]

Taylor SE, Gonzaga GC, Klein LC, Hu P, Greendale GA, Seeman TE. Relation of oxytocin to psychological stress responses and hypothalamic-pituitary-adrenocortical axis activity in older women. Psychosomatic Medicine. 2006; 68(2):238-245. [PubMed: 16554389]

Taylor SE, Klein LC, Lewis BP, Gruenewald TL, Gurung RA, Updegraff JA. Biobehavioral responses to stress in females: Tend-and-befriend, not fight-or-flight. Psychological Review. 2000; 107(3): 411-429. [PubMed: 10941275]

Uvnas-Moberg K. Role of efferent and afferent vagal nerve activity during reproduction: Integrating function of oxytocin on metabolism and behaviour. Psychoneuroendocrinology. 1994; 19(5-7): 687-695. [PubMed: 7938364]

Uvnas-Moberg K. Antistress pattern induced by oxytocin. News in Physiological Sciences. 1998a; 13:22-25. [PubMed: 11390754]

Uvnas-Moberg K. Oxytocin may mediate the benefits of positive social interaction and emotions. Psychoneuroendocrinology. 1998b; 23(8):819-835. [PubMed: 9924739]

Uvnas-Moberg K, Bruzelius G, Alster P, Lundeberg T. The antinociceptive effect of non-noxious sensory stimulation is mediated partly through oxytocinergic mechanisms. Acta Physiologica Scandinavica. 1993; 149(2):199-204. [PubMed: 8266809]

White-Traut R, Watanabe K, Pournajafi-Nazarloo H, Schwertz D, Bell A, Carter CS. Detection of salivary oxytocin levels in lactating women. Developmental Psychobiology. 2009; 51(4):367-373. [PubMed: 19365797]

Williams JR, Carter CS, Insel T. Partner preference development in female prairie voles is facilitated by mating or the central infusion of oxytocin. Annals of the New York Academy of Sciences. 1992; 652:487-489. [PubMed: 1626857]

Windle RJ, Brady MM, Kunanandam T, Da Costa AP, Wilson BC, Harbuz M, et al. Reduced response of the hypothalamo-pituitary-adrenal axis to alpha1-agonist stimulation during lactation. Endocrinology. 1997a; 138(9):3741-3748. [PubMed: 9275060]

Windle RJ, Wood S, Shanks N, Perks P, Conde GL, da Costa AP, et al. Endocrine and behavioural responses to noise stress: Comparison of virgin and lactating female rats during non-disrupted maternal activity. Journal of Neuroendocrinology. 1997b; 9(6):407-414. [PubMed: 9229351] 


\begin{tabular}{|c|c|c|c|c|c|c|c|c|}
\hline Saliva Samples & & & & & & & & \\
\hline $\begin{array}{c}\text { BASE } \\
7\end{array}$ & $\begin{array}{l}\text { INTXN } \\
5\end{array}$ & $\begin{array}{c}\text { POST-INTXN } \\
7\end{array}$ & $\begin{array}{c}\mathrm{SPC} \\
3\end{array}$ & $\begin{array}{c}\text { REP } \\
3\end{array}$ & $\begin{array}{c}\text { ICE } \\
2\end{array}$ & $\begin{array}{c}\text { RECOV } \\
7\end{array}$ & $\begin{array}{c}\text { FEED ad lib } \\
10\end{array}$ & $\begin{array}{l}\text { Feed } \\
\text { cont'd }\end{array}$ \\
\hline
\end{tabular}

Figure 1.

Timing of protocol and oxytocin sampling. Base: Baseline rest; INXN: 5-min mother-infant interaction; POST-INTXN: Post-interaction rest; SPC: Speech; REP: Speech replay; ICE: 2min forehead cold pressor; REC: Post-stress recovery; FEED: Infant feeding by breast or formula. 

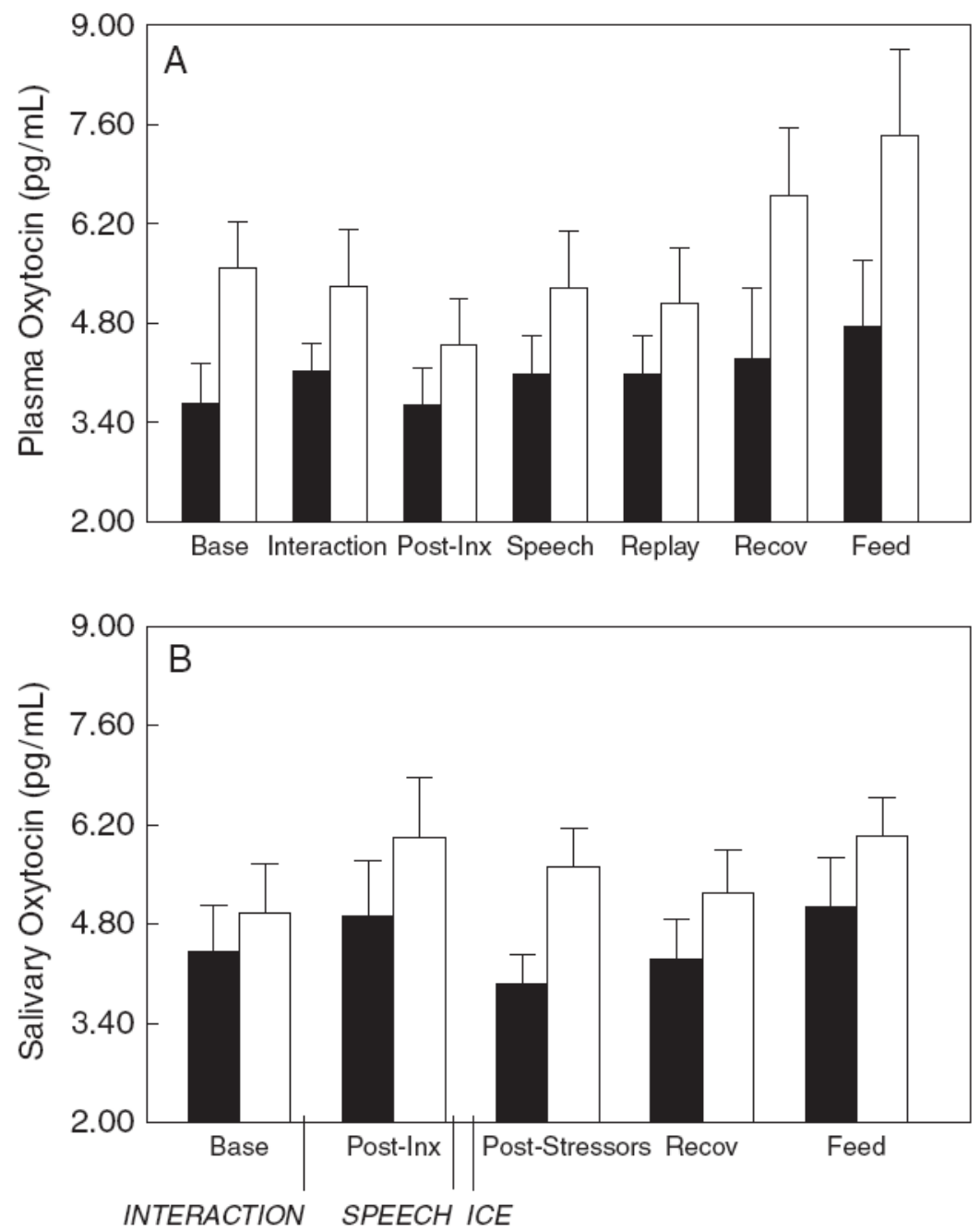

Figure 2.

Mean plasma (A) and salivary (B) oxytocin concentrations (pg/mL) assayed at different time points calculated for Breast and Formula-feeding mothers separately. Error bars depict standard error of the mean. ${ }^{*} p<.05$. 


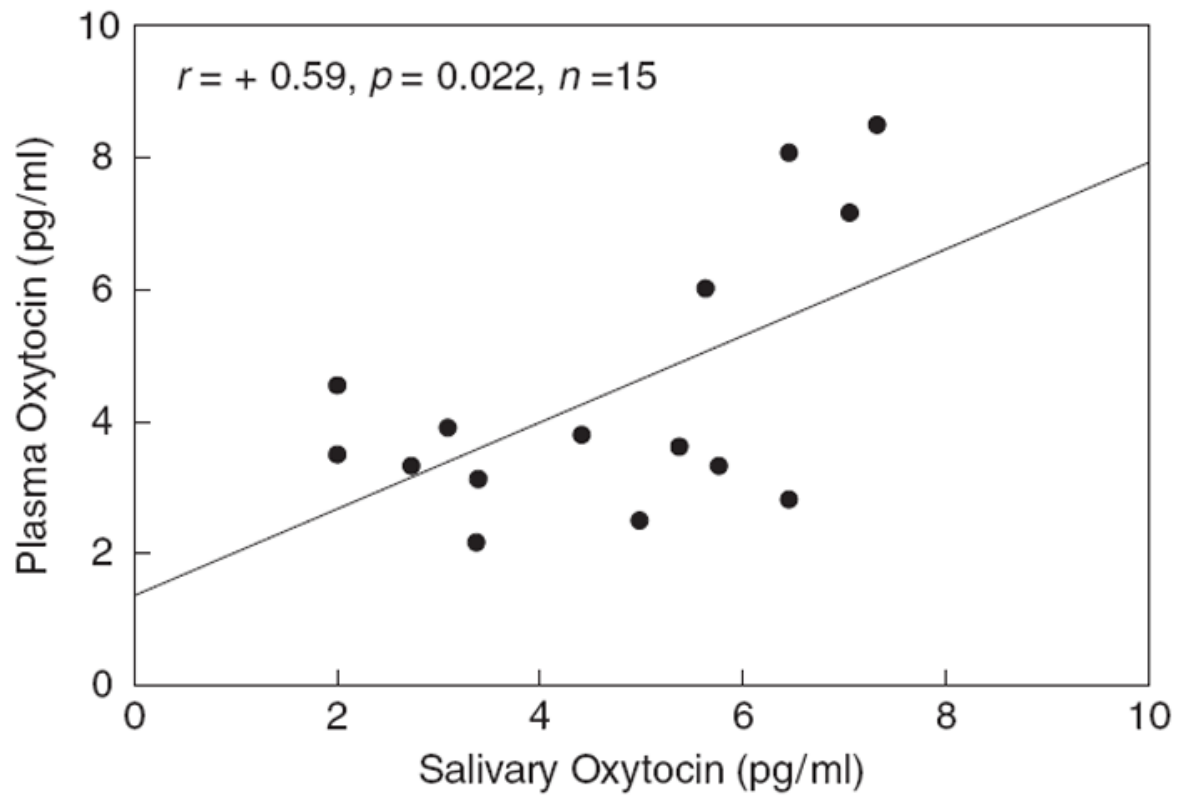

Figure 3.

Pearson correlations between plasma and salivary oxytocin concentrations (pg/mL) sampled at minute 7 of Resting Baseline. 


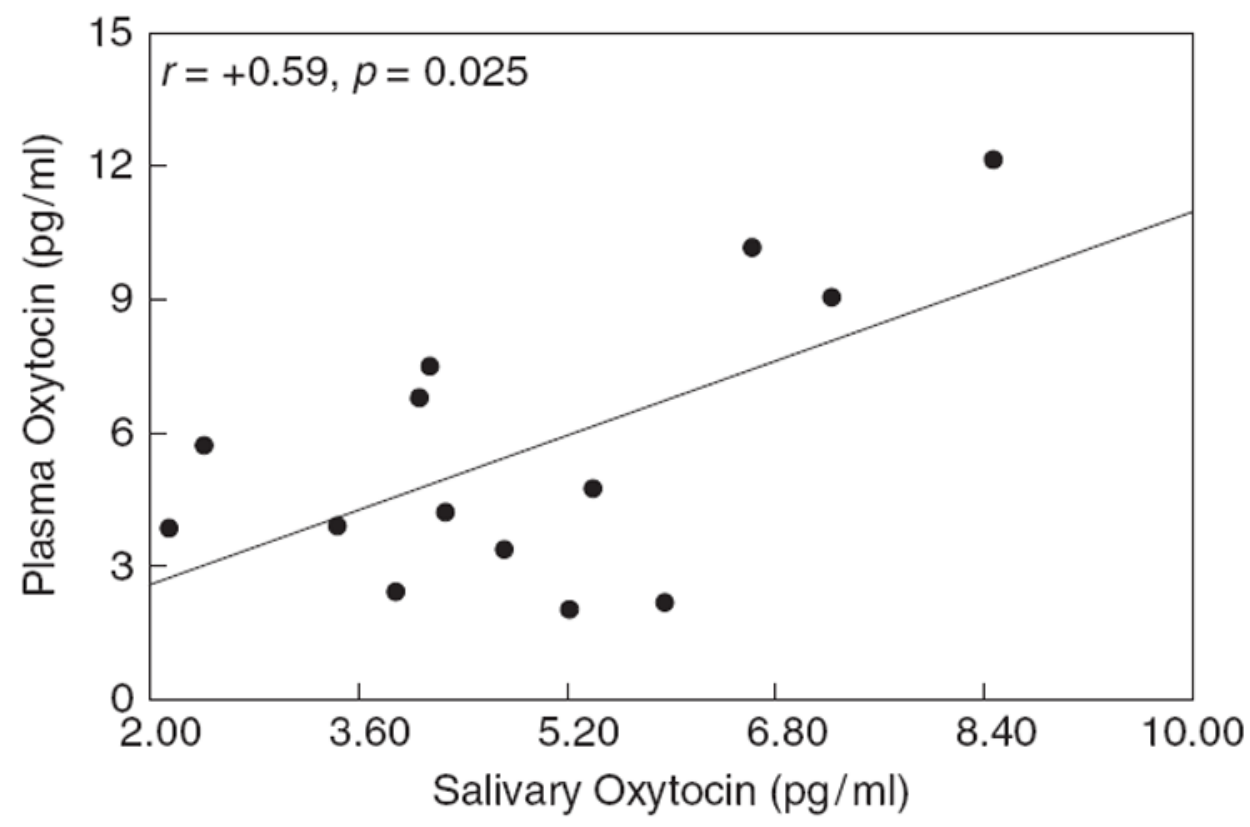

Figure 4.

Pearson correlations between plasma and salivary oxytocin concentrations (pg/mL) sampled at minute 7 of Resting Recovery following stressors. 


\section{Table 1}

Subject Characteristics

\begin{tabular}{lrcr}
\hline Variable & Mean & Std Err & Range \\
\hline Maternal age (years) & 30.80 & 1.40 & $21.0-45.0$ \\
Body mass index $\left(\mathrm{kg} / \mathrm{m}^{2}\right)$ & 27.15 & 1.10 & $18.6-39.1$ \\
Gestational age (weeks) & 39.35 & 0.45 & $34.0-41.9$ \\
Birth weight (pounds) & 7.04 & 0.32 & $4.9-10.0$ \\
Infant age at testing (days) & 55.85 & 4.80 & $30.0-96.0$ \\
\hline
\end{tabular}




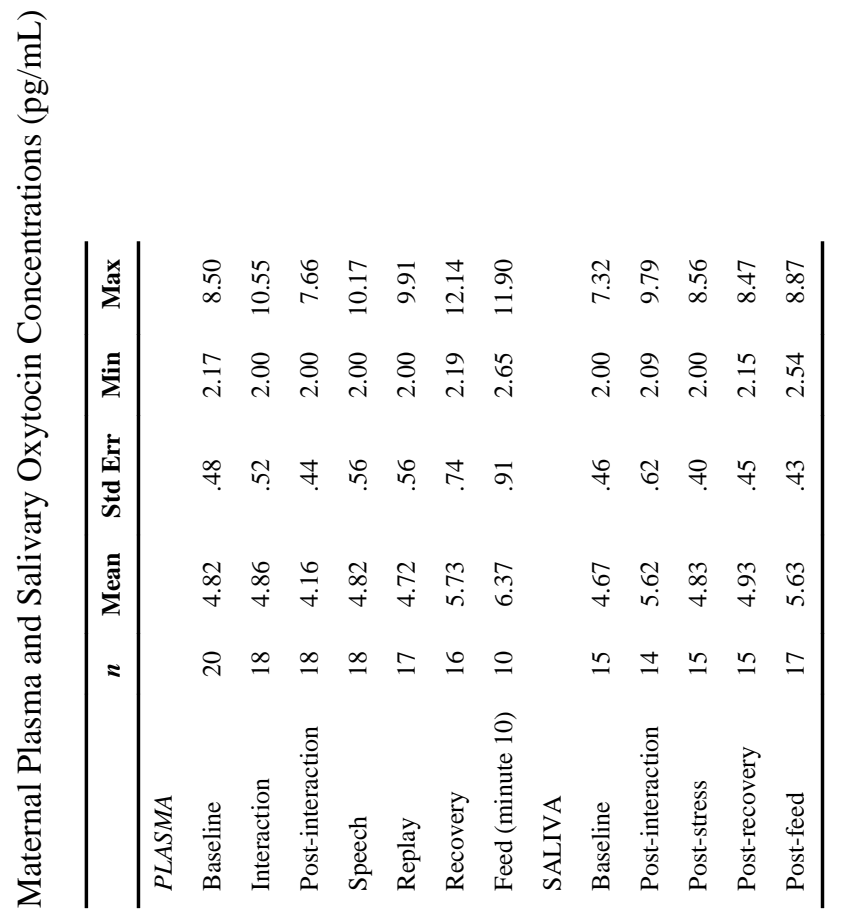

